\title{
Advances from Recent Multi-wavelength Campaigns on Sgr A*
}

\author{
Daryl Haggard \\ McGill Space Institute, McGill University Department of Physics \\ 3550 University Street, Montreal, QC, Canada \\ email: daryl.haggard@mcgill.ca
}

\begin{abstract}
Sagittarius A* is the closest example of a supermassive black hole and our proximity allows us to detect emission from its accretion flow in the radio, submillimeter, near IR, and Xray regimes. Ambitious monitoring campaigns have yielded rich multi-wavelength, time-resolved data that have the power to probe the physical processes underlying Sgr A*'s quiescent and flare emission. Here, I review the status of Sgr A* X-ray monitoring campaigns from the Chandra Xray Observatory (also XMM Newton, and Swift), and efforts to coordinate these with observations across the electromagnetic spectrum. I also discuss how these observations constrain models for Sgr A*'s variability, which range from tidal disruption of asteroids to gravitational lensing to collimated outflows to magnetic reconnection.
\end{abstract}

Keywords. Galaxy: center, black hole physics, accretion, accretion disks, galaxies: active

\section{Introduction}

The Milky Way's galactic nucleus is a hive of activity, hosting a supermassive black hole, Sagittarius A* $\left(\sim 4 \times 10^{6} \mathcal{M}_{\odot}\right.$; Ghez et al. 2008; Gillessen et al. 2009; Boehle et al. 2016), a massive star cluster (e.g., Ghez et al. 2008; Do et al. 2013), and many larger scale structures including the "minispiral" (e.g., Zhao et al. 2010), the circumnuclear disk (e.g., Lau et al. 2013), the Sgr A East supernova remnant (e.g., Zhao et al. 2016), gossamer radio filaments (e.g., Yusef-Zadeh et al. 2004; see also review by M. Morris in these proceedings), and cold molecular gas (see Fig. 1 and references therein).

In this short review, I focus on high-resolution observations of Sgr A* from the Chandra X-ray Observatory, and companion multi-wavelength campaigns. These observations reveal the supermassive black hole $(\mathrm{SMBH})$ during quiescence, when the X-ray emission directly probes the accretion flow and associated outflows, e.g., jets and winds (e.g., Wang et al. 2013). Both observations and models confirm that the SMBH captures its fuel from the winds of nearby massive stars (e.g., Cuadra et al. 2008; Wang et al. 2013; Russell et al. 2016). Multi-wavelength data also uncover high contrast variability in the form of $\mathrm{X}$-ray and multi-wavelength flares (see below), whose physical driver is still uncertain, though possible mechanisms include magnetic reconnection, shocks, tidal disruption of smaller bodies, and gravitational lensing. X-ray observations can also probe processes at larger scales, tracing dynamical interactions between members of the S-star cluster (e.g., Ghez et al. 2008; Do et al. 2013), hypervelocity stars (Zubovas et al. 2013; Rossi et al. 2016), and interloping low-mass objects like G2 (e.g., Gillessen et al. 2012). Sgr A* and its surroundings provide one of our best testbeds for strong gravity, with compelling upcoming observations by the Event Horizon Telescope poised to reveal the SMBH shadow (Doeleman et al. 2009; Fish et al. 2009), and to look for precession of S0-2's periapse during it's close approach in 2018 (Ghez et al. 2008). High energy observations, notably from Fermi and XMM Newton, but also from Chandra and other observatories, have 


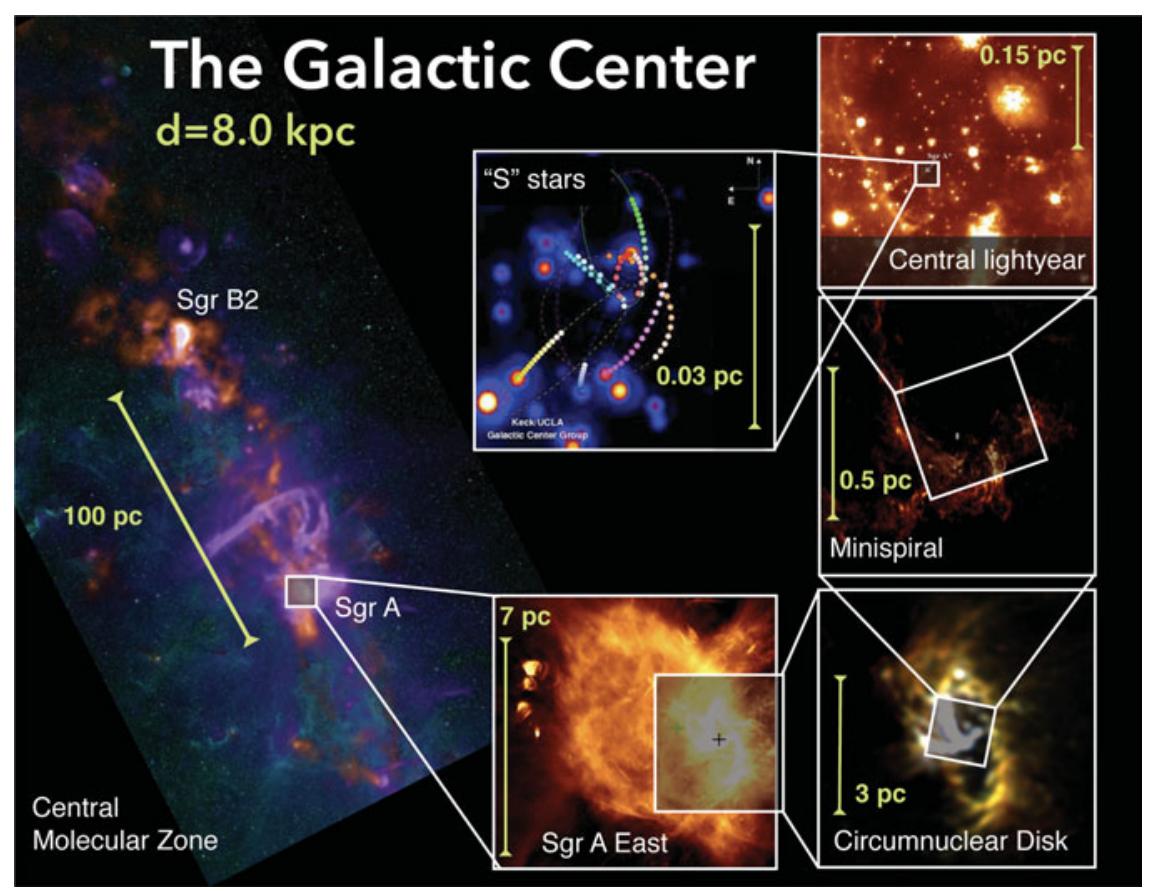

Figure 1. Counter clockwise from left to right: The central molecular zone (CMZ) showing synchrotron emission and ionized gas at $20 \mathrm{~cm}$ from VLA (purple; Yusef-Zadeh (2004)); 1.1 $\mathrm{mm}$ cold dust from CSO-Bolocam (orange; Bally (2010)); and stars and hot dust at $8 \mu \mathrm{m}$ from Spitzer-IRAC (teal; Stolovy (2006)). (CMZ composite image credit: A. Ginsburg [NRAO].) Synchrotron emission from the Sgr A East supernova remnant and ionized gas at $6 \mathrm{~cm}$ from VLA (Zhao (2016)). The Circumnuclear Disk (CND) traced via hot dust emission at 19.7, 31.5 and $37.1 \mu \mathrm{m}$ by SOFIA-FORCAST (Lau (2013)). Ionized gas and synchrotron emission from the Galactic Center "minispiral" resolved at $1.3 \mathrm{~cm}$ by VLA (Zhao (2010)). Stars and dust occupying the central lightyear, observed with Keck in the L-band $(3.8 \mu \mathrm{m}$; Ghez (2005)). Massive stars in the "S"-star cluster at $2.1 \mu \mathrm{m}$ (Keck L-band), with stellar orbits from the UCLA Galactic Center Group (Ghez (2008)). Montage courtesy of Betsy Mills (San Jose State University), reproduced with permission; color version available online.

picked up evidence for past activity from the black hole, including the Fermi bubbles and light echoes in Galactic Center molecular clouds (e.g., Su et al. 2010; Ponti et al. 2010).

In addition to tracing Sgr $\mathrm{A}^{*}$ 's highly variable behavior, X-ray monitoring campaigns from Chandra, XMM, and Swift have uncovered a large population of Galactic Center X-ray binaries (e.g., Degenaar et al. 2013; 2015). These include an assortment of black hole X-ray binaries and neutron star binaries, and the recent discovery of a young magnetar in the vicinity of the SMBH (Kennea et al. 2013; Mori et al. 2013; Rea et al. 2013). The magnetar has, in particular, opened a fresh debate on the composition of the stellar population in our Galaxy's center, and whether the stellar mass function has been impacted by the violent radiation fields permeating the region, and/or sculpted by dynamical processes (e.g., Dexter \& O'Leary 2014; Bower et al. 2015a).

\section{Intensive Multi-wavelength Campaigns}

The Chandra X-ray Visionary Project. Sgr A* was first confirmed as an X-ray source by Chandra, which detected it as a faint source (L $[2-10 \mathrm{keV}] \sim 2 \times 10^{33} \mathrm{erg} \mathrm{s}^{-1}$ ) with a size of $\sim 1^{\prime \prime}$ (Baganoff et al. 2003; Xu et al. 2006), at a distance of $\sim 8 \mathrm{kpc}$ (e.g., Boehle 
et al. 2016). Sgr A*'s quiescent emission arises from bremsstrahlung emission from a hot plasma ( $\mathrm{T} \sim 7 \times 10^{7} \mathrm{~K}, \mathrm{n}_{e} \sim 100 \mathrm{~cm}^{-3}$ ) emitted from a region very close to the event horizon (e.g., Melia 1992; Quataert 2002). Since this early discovery, the most ambitious X-ray campaign undertaken for Sgr A* to-date is the Chandra X-ray Visionary Project (XVP), which acquired 3 Msec of pointed observations with the Chandra High Energy Transmission Gratings between February and October 2012 (Chandra's cycle 13) $\dagger$. The Sgr A* XVP offers the first ever high resolution, time resolved X-ray spectra of Sgr $A^{*}$ for studies of accretion inflow/outflow, flares, Galactic Center diffuse emission and X-ray point sources. The Chandra observations were also coordinated with observations from Fermi, NuSTAR, XMM, Swift (daily), Hubble, Spitzer, Keck,VLT, ALMA, SMA, $V L A, V L B A$ and simultaneous monitoring was performed wherever possible.

Several pivotal studies have already emerged from the XVP, including results from Wang et al. (2013), which offers conclusive evidence for an accretion flow onto the SMBH. Earlier studies had not been able to distinguish between coronally active spun-up stars vs. accretion for the source of the quiescent X-ray emission immediately in the vicinity of Sgr A* (Baganoff et al. 2003; Sazonov et al. 2012). Wang et al. (2013) report a lack of Fe K- $\alpha$ in the deep X-ray spectra from Sgr A*, which rules out stars as the primary $\mathrm{X}$-ray source and argues in favor of accretion. The accretion hypothesis is also supported by the presence of optically thin He- and H-like lines ( $\mathrm{Si}, \mathrm{S}, \mathrm{Ar}$ ), which are predicted by radiatively inefficient accretion models. Wang et al. (2013) also report elongation in the quiescent emission, likely in the direction of the clockwise stellar disk (Paumard et al. 2006, Lu et al. 2009), and find that $99 \%$ of the accreted mass is subsequently lost to outflows.

These observational findings allow detailed comparisons to theoretical models and simulations, which constrain the plasma conditions and outflows (winds and jets) in this strong gravity environment around Sgr A* General relativistic models from Dibi et al. (2012) and Drappeau et al. (2013) incorporate optically thin $(\tau<1)$ cooling into models for the first time, and favor an accretion rate of $\sim 10^{-8} \mathcal{M}_{\odot} \mathrm{yr}^{-1}$ (Genzel et al. 2010), in agreement with earlier models evoking a radiatively inefficient accretion flow (e.g., Yuan et al. 2003, 2004). Multi-wavelength observations of Sgr A* also allow our Galaxy's BH to be placed into the context of other low- and high-mass accreting black holes, e.g., $\mathrm{X}$-ray binaries and low luminosity AGN. These comparative studies look for correlations between the jet-break frequency (indicating a transition to low $\tau$ ) and X-ray power law index (tracing the hot corona; e.g., see recent work by Koljonen, et al. 2015) and suggest an intrinsic connection between the plasma close to the $\mathrm{BH}$ and the outflow.

In addition to constraining accretion flow models, the XVP tripled the number of known X-ray flares, increasing it from roughly 20 to more than 60 , and demonstrating correlations between the flare durations, fluences, and peak luminosities (Neilsen et al. 2013). The HETG gratings eliminated X-ray pile up in Chandra observations for the first time, even for the brightest flares (Nowak et al. 2012, Neilsen et al. 2013). The $\mathrm{X}$-ray flares are high-contrast events, distinct from the quiescent X-ray emission, though faint X-ray flares are more common than bright ones, and it is likely that $\sim 10 \%$ of the quiescent emission may be attributed to unresolved, weak X-ray flares (Neilsen et al. 2013, 2015). Both the X-ray and IR flux distributions are consistent with a power law distribution and indicate that most of the flux $(80-90 \%)$ arises from steady quiescent emission consistent with a Poisson process (e.g., Dodds-Eden et al. 2011; Witzel et al. 2012; Meyer et al. 2014; Neilsen et al. 2015). Both X-ray and IR flares (or flickering) demonstrate short timescale variability, indicating that they emanate from a compact

$\dagger$ The official Sgr A* Chandra XVP webpage is: http://www.sgra-star.com/. 


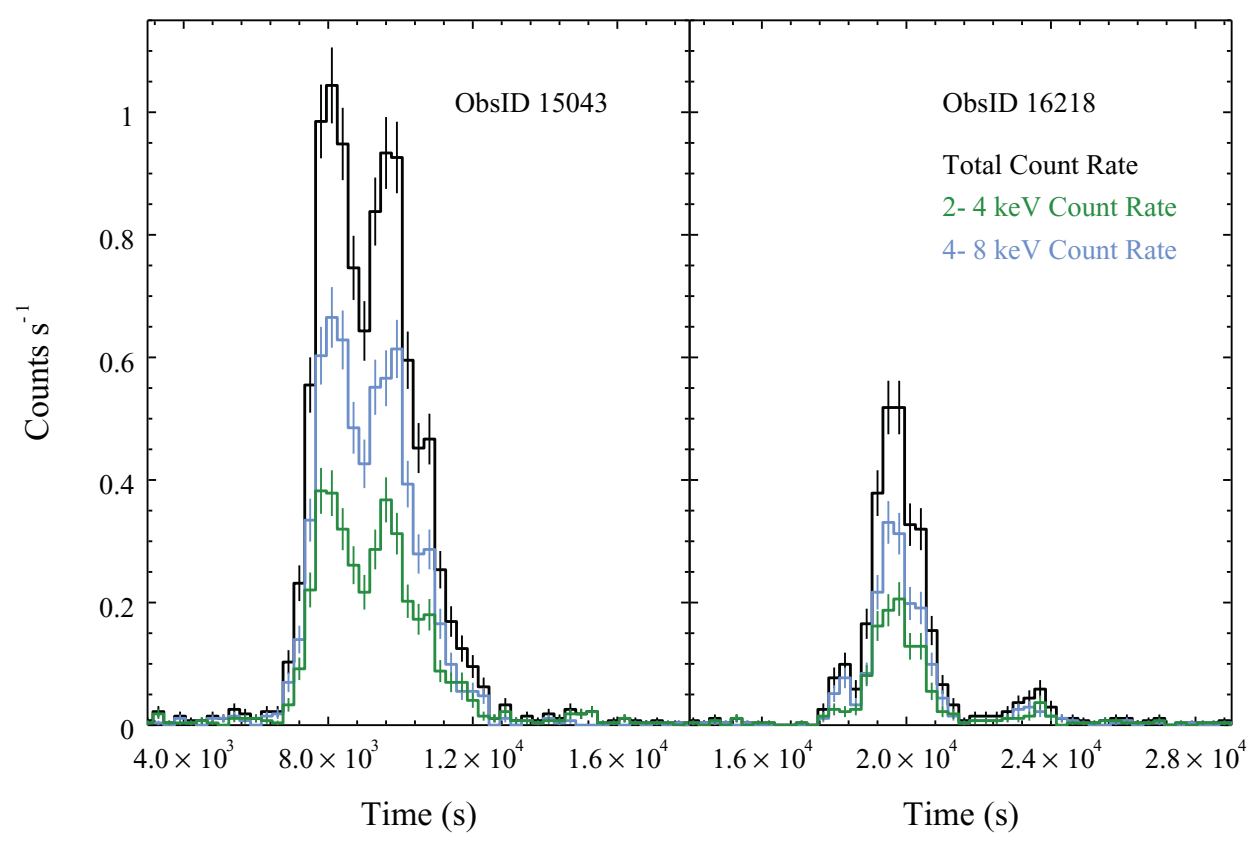

Figure 2. Light curves for two brightest Chandra X-ray flares detected during the 2013 and 2014 LP observing programs. The first was observed in September 2013 (left panel), before G2's pericenter passage, the second was observed in October 2014 (right panel), soon after the Sgr A*/G2 closest encounter. The upper-most (black) line marks the total Chandra 2-10 keV count rate $\left(\right.$ counts $\mathrm{s}^{-1}$ ) vs. time (s), the middle (light blue) line indicates the "hard" $4-8 \mathrm{keV}$ rate, and the bottom (green) line traces the "soft" $2-4 \mathrm{keV}$ rate. There is no strong evolution in the hardness ratio as a function of time during these bright flaring events. Figure from Haggard et al., in preparation; a color version of this figure is available online.

region, e.g., within $\sim 10$ Schwarzschild radii of Sgr A* (Barriere et al. 2014), and may arise from the same population of electrons (e.g., see Hornstein et al. 2007 and Ponti et al., in prep).

Sgr A*/G2 Monitoring Campaigns. Subsequent to the Chandra XVP program in 2012, several Chandra large programs (LPs) and target of opportunity ( $\mathrm{ToO}$ ) proposals were launched to continue Galactic Center monitoring at X-ray and other complementary wavelengths. These programs tracked the $\mathrm{SgrA}^{*} / \mathrm{G} 2$ interaction (Gillessen et al. 2012; Phifer et al. 2013; Pfuhl et al. 2015) and the newly-discovered magnetar (e.g., Coti Zelati et al. 2015, and references therein), and continued to search for and characterize Sgr A* flares. The Sgr A*/G2 and magnetar programs, in particular, were coordinated with massive simultaneous observational campaigns from Fermi, NuSTAR, XMM Newton, Swift (daily), Hubble, Spitzer, Keck, VLT, ALMA, SMA, VLA, and VLBA, among others†.

\section{Bright X-ray Flares}

In addition to the large, statistical sample of flares discovered via the Chandra XVP, the two Chandra LPs undertaken in 2013 and 2014 uncovered additional very bright flares, including the two brightest flares ever detected. These rise 200 to 400 times above the quiescent X-ray background (Figure 2; Haggard, et al. 2014; Ponti et al. 2015; Haggard,

$\dagger$ For a recent popular review, see the cover article for the February 2016 issue of Sky $\mathscr{E}$ Telescope magazine, by D. Haggard and G. Bower; http://msi.mcgill.ca/MilkyWayCenter.pdf. 
Table 1. Spectroscopic Comparison of Bright X-ray Flares from Sgr A*.

\begin{tabular}{|c|c|c|c|c|c|c|c|c|}
\hline Flare & Obs. & {$\left[10^{23^{\mathbf{N ~ H}}} \mathrm{cm}^{-2}\right]$} & $\Gamma$ & $\begin{array}{r}\mathbf{F} \\
{[10-12}\end{array}$ & $\begin{array}{l}\left(2-8 \mathrm{keV}^{-}\right. \\
\left.\text {erg } \mathrm{cm}^{-2} \mathrm{~s}^{-1}\right]\end{array}$ & $\begin{array}{l}\text { Dur. } \\
{[\mathrm{ks}]}\end{array}$ & $\begin{array}{c}\text { F luence } \\
{\left[10^{-8} \text { erg } \mathbf{c m}^{-2}\right]}\end{array}$ & $\begin{array}{c}E(2-10 \mathrm{keV}) \\
{\left[10^{38} \mathrm{erg}\right]}\end{array}$ \\
\hline Haggard + in prep $^{1}$ & Chandra & $\begin{array}{c}1.43+0.69 \\
-1.5\end{array}$ & $\begin{array}{r}2.1+0.1 \\
-0.3 \\
\end{array}$ & & $\begin{array}{r}21+0.4 \\
-0.3 \\
\end{array}$ & 6.6 & $14 \pm 0.3$ & 17 \\
\hline Nowak +12 & Chandra & $\begin{array}{r}1.43_{-3.6}^{+4.4} \\
\end{array}$ & $\begin{array}{r}2.0+0.7 \\
-0.6\end{array}$ & & $8.5 \pm 0.9$ & 5.6 & $4.7 \pm 0.5$ & 10 \\
\hline $\begin{array}{l}\text { Porquet }+08, \\
\text { Nowak }+12^{2}\end{array}$ & $\begin{array}{c}\text { X M M } \\
\text { Newton }\end{array}$ & $\begin{array}{r}1.63_{-2.6}^{+3.0} \\
-2.6\end{array}$ & $2.4_{-0.3}^{+0.4}$ & & $\begin{array}{r}4.8^{+0.2} \\
-0.3\end{array}$ & 2.9 & $1.4 \pm 0$ & 3.5 \\
\hline $\begin{array}{l}\text { Porquet }+08, \\
\text { Nowak }+12^{2}\end{array}$ & $\begin{array}{c}\text { X M M } \\
\text { Newton }\end{array}$ & $\begin{array}{r}1.61_{-2.2}^{+1.9}\end{array}$ & $2.3 \pm 0.3$ & & $7.7 \pm 0.3$ & 2.8 & $2.2 \pm 0.1$ & 5.3 \\
\hline
\end{tabular}

Notes:

${ }^{1}$ Preliminary spectroscopic fit for the September 2013 Chandra flare (Haggard et al., in preparation).

${ }^{2}$ XMM Newton bright X-ray flares from Porquet et al. (2008); spectroscopic fits from re-analysis presented in Nowak et al. (2012).

et al. in prep) - the previous record-holder from the XVP was reported by Nowak et al. (2012), and reached $\sim 140 \times$ the quiescent level. I outline a preliminary study of the bright 2013 flare here, and refer readers to Haggard et al., in preparation, for a detailed analysis of both the 2013 and 2014 bright flares.

The Brightest Known Chandra X-ray Flare. In September 2013, Chandra detected the brightest X-ray flare ever observed (Figure 2, left panel). This flare lasted approximately $\sim 6.0 \mathrm{ks}$, has a mean count rate of 0.41 counts $\mathrm{s}^{-1}$, and a total fluence of $\sim 2500$ counts, corresponding to a total $2-10 \mathrm{keV}$ emission energy of $\sim 2 \times 10^{39} \mathrm{erg}$ (Table 1 ). This flare is brighter and shows a longer duration than brightest flares in the Chandra XVP sample, though it agrees with the trend toward longer durations at higher luminosities (Neilsen et al. 2013). Preliminary modeling of the X-ray spectrum for flare-only intervals reveals a fit consistent with a power law $(\Gamma \sim 2.1)$, for a column density of $\mathrm{NH} \sim 1.43 \times 10^{23} \mathrm{~cm}^{-2}$, consistent with fits reported for several bright flares previously detected by Chandra and XMM (Table 1; Porquet et al. 2008; Nowak et al. 2012).

This bright flare also has a unique double-peaked morphology. Detailed analysis of the flare's morphology and time series are enabled by the Chandra instrument configuration during these observations, which include a $1 / 8$ subarray on a single ACIS-S detector, giving timing resolution as fine as 0.4 seconds. Early analysis of the flare power spectral density indicates evidence for excess power above random noise on timescales of 150 seconds and longer. These timescales are interesting in the context of predicted quasiperiodic oscillations (QPOs) for Sgr $\mathrm{A}^{*}$ in general relativistic magneto?hydrodynamic (GRMHD) simulations (e.g., Dolence et al. 2012), particularly near the innermost stable circular orbit (ISCO) orbital frequency at IR and X-ray wavelengths (Haggard, et al. in prep).

Detailed timing studies of Sgr A*'s bright flares may help disentangle some of the lingering uncertainties about the physical mechanism that drives these high-contrast Xray events. We know from studies like Neilsen et al.(2013) and others that bright flares typically last a few thousand seconds. These timescales are consistent with the orbital period of material at the radius of the ISCO, and also with the characteristic flyby time for asteroids at $1 \mathrm{AU}$, as well as the Alfvén crossing time for magnetic loops, and possibly even the timescales for gravitational lensing of magnetically confined flux tubes (see "Flare Models" later in this section). Tighter constraints on any characteristic subflare times scales, as well as the rise and fall times for the flares, may help distinguish between these possible flare origins. 


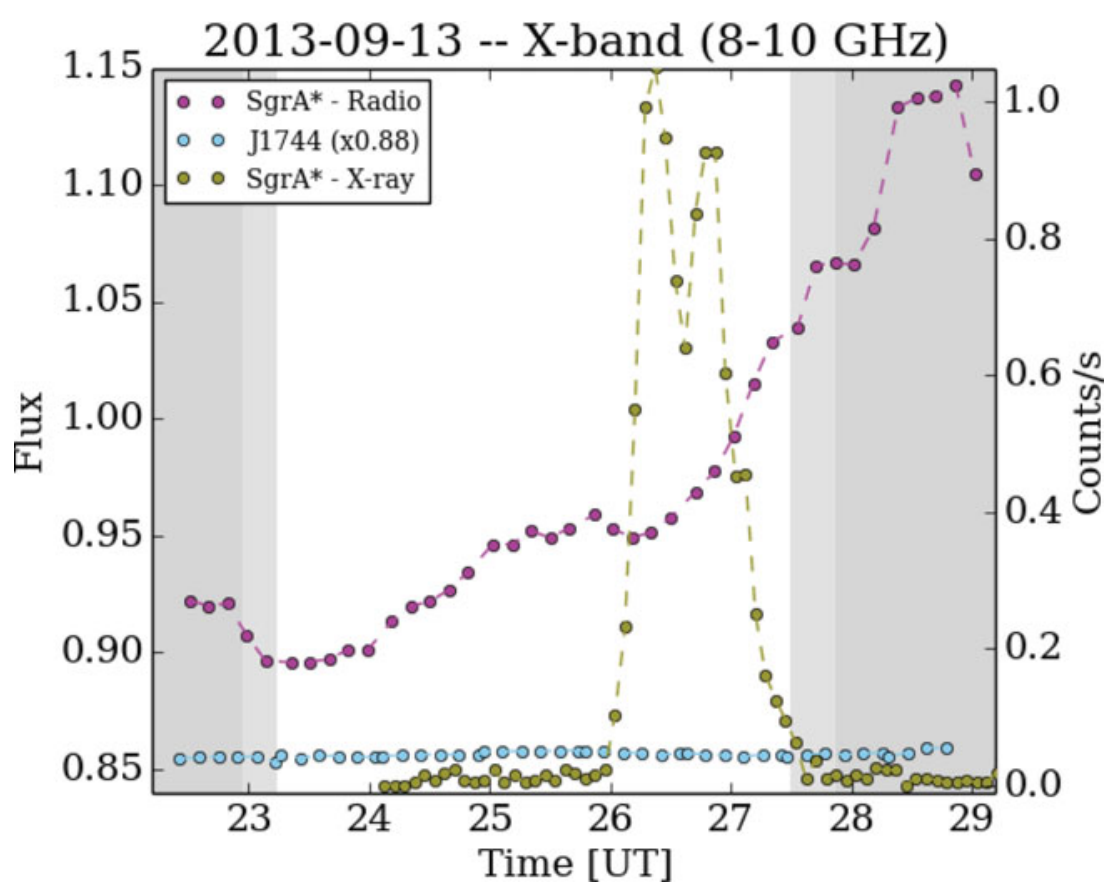

Figure 3. Chandra X-ray (green) and VLA radio (purple) lightcurves for the September 2013 bright flare, in addition to the radio phase calibrator, J1744 (cyan). The left vertical axis shows the $8-10 \mathrm{GHz}$ radio flux (average flux per scan over all antennae and SPW for baselines longer than $50 \mathrm{k} \lambda$ ), while the right vertical axis indicates the $2-10 \mathrm{keV} \mathrm{X}$-ray count rate (see also Figure 2), and the horizontal axis is time in UT hours. Darker gray shaded regions denote time intervals where both Sgr A* and J1744 are below 18 degrees on the sky; the light shaded regions mark where only J1744 is below 18 degrees. Figure from Capellupo et al., in preparation; a color version of this figure is available online.

Simultaneous multi-wavelength observations offer another constraint that may be crucial in breaking model degeneracies. For the bright September 2013 X-ray flare, we obtained simultaneous, continuous VLA coverage (Figure 3). These $3.6 \mathrm{~cm}(8-10 \mathrm{GHz})$ radio observations show a flux increase of $\sim 25 \%$ with a cross correlation peak at $>130$ minutes, consistent with previous time delay estimates for Sgr A* flares (e.g., Brinkerink et al. 2015). There is also a hint of an anti-correlation between the radio and the X-ray just as the X-ray reaches its peak.

The Sgr A*/G2 Encounter. In early 2012, Gillessen et al. reported a cold, extended object whizzing toward Sgr A* on a nearly radial orbit, and nicknamed it G2. The object's closest approach was initially expected in summer 2013 (later revised to spring 2014), with a distance $\sim 2200$ times the event horizon ( $<150 \mathrm{AU})$. G2 was detected in M and L' (far-IR), but not Ks or H (near-IR), photometry and showed distinctive IR emission lines (e.g., Brackett- $\gamma$ and Paschen- $\alpha$; Gillessen et al. 2012; Pfuhl, et al. 2015, and references therein). Early works posited that G2 was a cold, 3 Earth-mass gas cloud, and that it's encounter with the SMBH might ignite shocks or an outburst in "real time" as G2's material was tidally stripped and fell onto the black hole. Soon, however, a debate arose over whether G2 was a cloud or an embedded low-mass star (e.g., Phifer et al. 2013; Witzel, et al. 2014; Sitarski, B. et al. in preparation); this debate is not fully resolved even today. 
In this context, Chandra and other observatories trained their "eyes" on the Galactic Center, searching for a hint (beyond the IR) of the Sgr A*/G2 interaction. In the $~ 900 \mathrm{ks}$ of Chandra X-ray data collected by our team in 2013 and 2014, no rise in the underlying Xray flux from Sgr A* was observed (Haggard et al. 2014). There was also no clear increase in Sgr A*'s radio emission (Bower et al. 2015b). These X-ray and radio observations indicate that no shock front formed as G2 plunged through the hot ambient medium around Sgr A*. This could be due to clumpiness in either the accretion flow or in the G2 object itself, or it could be that G2's predecessor, G1, had already cleared their mutual orbital path, e.g., if G1 and G2 are both remnants from an earlier tidal disruption of a massive star (Guillachon et al. 2015). Another, perhaps more likely, possibility is that initial estimates of the properties of the accretion flow were incorrect, e.g., the pressure and density of the accretion flow may be lower than expected. Certainly, the viscosity of the flow and hence the accretion timescales (which vary from months to years, depending on the assumed parameters) are not well known. Hence, the non-detection of G2 is itself beginning to place constraints on both the accretion environment and on the nature of the G2 object (e.g., Morsony et al. 2015, Madigan et al. 2016).

In addition to increases in the X-ray and radio flux from Sgr $A^{*}$ 's quiescent flow and shock heating of G2 itself, monitoring campaigns searched for an increase in the X-ray flare rate, which could be catalyzed by a new source of infalling material from the passing object or from a perturbation associated with its passage. There is little evidence for an increase in the flare rate from the Chandra LP campaigns, but Ponti et al. (2015) analyze all of the Chandra, XMM Newton, and Swift X-ray data over the last $\sim 15$ years and find that there may be an increase in the number of bright flares near the G2 pericenter passage. (Note that XMM and Swift cannot access the faintest flares from Sgr A* after spring 2013 due to the magentar X-ray outburst.) They note, however, that this increase could also come from flare clustering. Indeed, Yuan \& Wang (2016) analyze archival Chandra data from 1999 - 2012 and find that faint flares cluster on timescales of $\sim 20-70 \mathrm{ks}$. These authors also note that there was no detectable increase in Sgr A*'s flare rate connected to S-02's last passage in $\sim 2002$. It will be worthwhile to check this constraint during S-02's next closest passage in 2018. An additional, joint campaign with $N u S T A R$ and $X M M$ also detects 3 bright flares within $\sim 1$ day (Barriere et al. 2014; Zhang et al. 2016), supporting the notion that bright flares may be detected in clusters.

Flare Models. With flares as one of the most interesting markers of Sgr A*'s activity, the question remains: what is the physical mechanism driving the flares? Two of the leading models are particle acceleration, e.g., via magnetic reconnection and/or shocks, and tidal disruptions, e.g., disruption of asteroids and planetesimals (Markoff et al. 2001; Liu \& Melia 2002; Liu et al. 2004; Yuan et al. 2003, 2004; Eckart et al. 2004, 2006; Marrone et al. 2008; Cadez et al. 2008; Kostic et al. 2009; Dodds-Eden et al. 2009; Yuan et al. 2009; Zubovas et al. 2012; Witzel et al. 2012; Yusef-Zadeh et al. 2012; Nowak et al. 2012; Neilsen et al. 2013; Dibi et al. 2014, 2016). A third interesting possibility has been posited recently by Ball et al. (2016). In their model, Sgr A*'s underlying variability and the time lags between wavelengths are driven by short-lived magnetic flux tubes which experience strong-field gravitational lensing near the horizon. In this model, the high contrast X-ray flares require additional electron injection, but ongoing GRMHD simulations might point to a reasonable mechanism, e.g., magnetic reconnection, in the near future.

Intensive studies of the IR and X-ray flux distributions, along with simultaneous IR/Xray flares, are also beginning to support detailed comparison between electron acceleration models, with synchrotron and synchrotron self Compton (SSC) mechanisms under 
the most scrutiny (e.g., Neilsen et al. 2013,2015; Witzel et al. 2012; Dibi et al. 2016; Fazio et al. in prep; Ponti et al. in prep). Modeling by Dibi et al. (2016) shows the IR flux cumulative distribution function (CDF) can constrain the distribution of acceleration slope fluctuations with synchrotron emission, while the X-ray CDF constrains distributions of the correlated magnetic field and flow density variations with SSC. Analysis of simultaneous multi-wavelength observations of individual flares are often stymied by incomplete coverage between wavelengths (though see new works coming soon from Fazio et al., in preparation, and Ponti et al., in preparation). To resolve these tensions, we need more simultaneous observations, in particular of bright radio/submm/IR/X-ray flares.

\section{Ongoing/Upcoming Multi-wavelength Campaigns}

To further advance studies of Sgr A*'s accretion and flaring activity, a number of observation campaigns are underway. One of the most ambitious (with the second set of observations arriving during this IAU symposium!) is a joint Spitzer and Chandra monitoring campaign (PI: Fazio) $\dagger$. This program has acquired two 24-hour, simultaneous Spitzer IRAC (4.5 micron) and Chandra $(2-10 \mathrm{keV})$ observations of Sgr A* in July 2016, with two additional epochs approved for July 2017. (The first set of Spitzer Sgr A* observations are reported in Hora et al. 2014.) Many partner telescopes are observing alongside this campaign, including (but not limited to) the $V L A, A L M A, S M A$, $V L T$ and Keck. These long, simultaneous light curves will enable detailed studies of the multi-wavelength flux distributions, as well as unambiguous association of IR and Xray variability and flares, lag times, etc. In addition, during Chandra's cycles's 17 and 18 both ToO (PI: Haggard) and instrument team (GTO, PI: Garmire) time have been allocated to Galactic Center observations. The Swift TAC has approved a key project to allow ongoing, daily observations $(1 \mathrm{ks})$ of the Galactic Center (PI: Degenaar), and NuSTAR has designated Galactic Center monitoring a key project. Frequent VLA ToOs are also approved for Sgr A* and Galactic Center X-ray transients. Meanwhile, the Event Horizon Telescope is nearing full operations (highlighted elsewhere in this proceedings) and will provide an unprecedented high-resolution view of the Sgr A* and it's accretion flow.

\section{Summary}

Recent multi-wavelength observational campaigns targeting Sgr A* offer new, tight constraints on the accretion and plasma conditions in the strong gravity regime. Wang et al. (2013) have used the Chandra XVP data to confirm a substantial outflow from Sgr $\mathrm{A}^{*}$; these data have also enabling detailed multi-wavelength flare studies (Nielsen et al. 2013). Subsequent Chandra campaigns have revealed the brightest flares yet detected (Ponti et al. 2015; Haggard et al., in prep). These have X-ray spectra comparable to other bright flares, but show their own unique properties, including asymmetric morphologies. Ongoing analysis will extract the detailed timing properties of these brightest flares, as well as investigate a likely lag between radio and X-ray flare emission.

To date, there has been no X-ray or radio sighting of the G2 object or its interaction with Sgr A*. Some works have hinted at a possible increase in Sgr A*'s bright flare rate, associated with G2's pericenter passage (Ponti et al. 2015), but this phenomena is also attributable to flare clustering, which seems to be a generic property of the fainter

$\dagger$ Details on the Spitzer/Chandra campaigns: https://www.cfa.harvard.edu/irac/gc/. 
flare population (Neilsen et al. 2013; Yuan \& Wang 2016). Detailed flux distributions (Witzel et al. 2013; Neilsen et al. 2015) and simultaneous multi-wavelength observations of bright, high S/N flares (Haggard et al. in prep; Ponti et al., in prep) are informing a variety of new GRMHD simulations (e.g., Dibi et al. 2016; Ball et al. 2016) and, though the flare mechanism and microphysics are still highly debated, theory and observations are nearing similar levels of precision, which point to a promising future.

\section{References}

Baganoff, F. K., et al. 2001, Nature, 413, 45

Baganoff, F. K., et al. 2003, ApJ, 591, 891

Ball, D., et al. 2016, ApJ, 826, 77

Bally, J., et al. 2010, ApJ, 721, 137

Barriere, N. M., et al. 2014, ApJ, 786, 46

Boehle, A., et al. 2016, ApJ accepted, arXiv:1607.05726

Bower, G. C., et al. 2015a, ApJ, 798, 120

Bower, G. C., et al. 2015b, ApJ, 802, 69

Brinkerink, C. D., et al. 2015, A $\mho A A, 576$, A41

Cadez, A., et al. 2008, A\&A487, 527

Capellupo, D., et al., in preparation

Coti Zelati, F., et al. 2015, MNRAS, 449, 2685

Cuadra, J., et al. 2008, MNRAS, 383, 458

Degenaar, N., et al. 2013, ApJ, 769, 155

Degenaar, N., et al. 2015, JHEA, 7, 137

Dexter, N., et al. 2014, ApJ, 783, 7

Dibi, S., et al. 2012, MNRAS, 426.1928

Dibi, S., et al. 2014, MNRAS, 441, 1005

Dibi, S., et al. 2016, MNRAS, 461, 552

Do, T., et al. 2013, MNRAS, 779, 6

Doeleman, S. S., et al. 2009, ApJ, 695, 59

Dodds-Eden, K., et al. 2009, ApJ, 698, 676

Dodds-Eden, K., et al. 2011, ApJ, 728, 37

Dolence, J. C., et al. 2012, ApJ, 746,10

Drappeau, S., et al. 2013, MNRAS, 431, 2872

Eckart, S., et al. 2004, A\&SA, 427, 1

Eckart, S., et al. 2006, A\&A A, 450, 535

Fazio, G., et al., in preparation

Fish, V. L., et al. 2009, ApJ, 692, L14

Genzel, R., et al. 2010, Reviews of Modern Physics, 82, 3121

Ghez, A. M., et al. 2004, ApJ, 601, 159

Ghez, A. M., et al. 2005, ApJ, 620, 744

Ghez, A. M., et al. 2008, ApJ, 689, 1044

Gillessen, S., et al. 2009, ApJ, 692, 1075

Gillessen, S., et al. 2012, Nature, 481, 51

Guillochon, J., et al. 2014, ApJ, 786, 12

Haggard, D., et al. 2014, Atel \#6242

Haggard, D., et al., in preparation

Haubois, X., et al. 2012, A\& A, 540, A41

Hora, X., et al. 2014, ApJ, 793, 120

Hornstein, S. D., et al. 2007, ApJ, 667, 900

Kennea, J. A., et al. 2013, ApJ, 770, 24

Koljonen, K. I. I.., et al. 2015, ApJ, 814,139

Kostic, U., et al. 2009, A\&A, 496, 307 
Lau, R. M., et al. 2013, ApJ, 775, 37

Liu, S. \& Melia, F. 2002, ApJ, 566, 77

Liu, S., Petrosian, V., \& Melia, F. 2004, ApJ, 611, 101

Lu, J. R., et al. 2009, ApJ, 690, 1463

Madigan, A.-M.; McCourt, M.; \& O'Leary, R. 2016, arXiv:1602.02760

Markoff, et al. 2001, A\&\&A, 379, L13

Marrone, et al. 2008, ApJ, 682, 373

Melia, et al. 1992, ApJ, 387, L25

Meyer, et al. 2014, ApJ, 791, 24

Mori, et al. 2013, ApJ, 770, L23

Morsony, B., et al. 2015, arXiv:1508.00384

Neilsen, J., et al. 2013, ApJ, 774, 42

Neilsen, J., et al. 2015, ApJ, 799, 199

Nowak, M. A., et al. 2012, ApJ, 759, 95

Paumard, T., et al. 2006, ApJ, 643, 1011

Pfuhl, O., et al. 2015, ApJ, 798, 111

Phifer, K., et al. 2013, ApJ, 773, 13

Ponti, T., et al. 2010, ApJ, 714, 732

Ponti, T., et al. 2015, MNRAS, 454, 1525

Ponti, G., et al., in preparation

Porquet, T., et al. 2003, A\&AA, 407, L17

Porquet, T., et al. 2008, A\&A A, 488, 549

Quataert, E., et al. 2002, ApJ, 575, 855

Rea, N., et al. 2013, ApJ, 775, L34

Russell, C. M., et al. 2016, arXiv:160701562

Rossi, E. M., et al. 2016, arXiv:160802000

Sazonov, S., et al. 2012, MNRAS, 420, 388

Sitarski, B., et al., in preparation

Stolovy, S., et al. 2006, JPhCS, 54, 176

Su, M., Slatyer, T. R., \& Finkbeiner, D. 2010, ApJ, 724, 1044

Wang, Q. D., et al. 2013, Science, 341, 981

Witzel, G., et al. 2012, ApJS, 203, 18

Witzel, G., et al. 2014, ApJ, 796, 8

Xu, Y.-D., et al. 2006, ApJ, 640, 319

Yuan, F., et al. 2003, ApJ, 598, 301

Yuan, F., et al. 2004, ApJ, 606, 894

Yuan, F., et al. 2009, ApJ, 703, 1034

Yuan, F. \& Wang, Q. D. 2016, MNRAS, 456, 1438

Yusef-Zadeh, F., et al. 2004, ApJS, 155, 421

Yusef-Zadeh, F., et al. 2012, AJ, 144, 1

Zhang, S., et al., in preparation

Zhao, J-.H., et al. 2010, ApJ, 723, 1097

Zhao, J-.H., Morris, M. R., \& Goss, W. M. 2016, ApJ, 817, 171

Zubovas, K., et al. 2012, MNRAS, 421, 1315

Zubovas, K., et al. 2013, ApJ, 771, 118 